\title{
Bifidobacterium Species Colonization in Infancy: A Global Cross-Sectional Comparison by Population His- tory of BreastFeeding
}

\author{
Diana H. Taft ${ }^{1,2}$, Zachery T. Lewis ${ }^{1 \sharp}$, Nhu Nguyen ${ }^{1}$, Steve Ho ${ }^{1}$, Chad Masarweh ${ }^{1}$, Vanessa Dunne- \\ Castagna $^{1}$, Daniel J. Tancredi ${ }^{3}$, M. Nazumul Huda ${ }^{4,5}$, Charles B. Stephensen ${ }^{4,5}$, Katie Hinde ${ }^{6}$, Erika \\ von Mutius ${ }^{7,8}$, Pirkka Kirjavainen ${ }^{9,10}$, Jean-Charles Dalphin ${ }^{11}$, Roger Lauener ${ }^{12}$, Josef Riedler ${ }^{13}$, Jennifer \\ T. Smilowitz ${ }^{1,2}$, J. Bruce German ${ }^{1,2}$, Ardythe L. Morrow ${ }^{14 *}$ and David A. Mills ${ }^{1,15^{*}}$
}

1 Department of Food Science and Technology, University of California Davis, Davis, CA, USA

2 Foods for Health Institute, University of California Davis, Davis, CA, USA

3 Department of Pediatrics, University of California Davis School of Medicine, Sacramento, CA, USA

4 US Department of Agriculture, Western Human Nutrition Research Center, Davis, CA, USA

5 Department of Nutrition, University of California Davis, Davis, CA, USA

6 Center for Evolution and Medicine, School of Human Evolution and Social Change, Arizona State University, Tempe, AZ, USA

7 Dr von Hauner Children's Hospital, Ludwig Maximilian University, Munich, Germany

8 Institute for Asthma and Allergy Prevention, Helmholtz Centre Munich, Germany

9 Environment Health Unit, National Institute for Health and Welfare, Kuopio, Finland

10 Institute of Public Health and Clinical Nutrition, University of Eastern Finland, Kuopio, Finland

11 University of Besançon, Department of Respiratory Disease, UMR/CNRS 6249 Chrono-environment, University Hospital, Besançon, France

12 Christine Kühne-Center for Allergy Research and Education, Davos, Switzerland

13 Children's Hospital Schwarzach, Austria

14 Department of Environmental and Public Health Sciences, University of Cincinnati, Cincinnati, OH, USA

15 Department of Viticulture and Enology, University of California Davis, Davis, CA, USA

* Correspondence: damills@ucdavis.edu(D.A.M.), Tel.: (530)754-7821; morrowa@ucmail.uc.edu(A.L.M.), Tel.: 26 (513)558-0809

\#Zachery T. Lewis now employed by Synbiotic Health, Lincoln, NE, USA

‡Deceased

\begin{abstract}
Bifidobacterium are a beneficial and dominant member of the breast-fed infant gut microbiome. However, the health benefits of Bifidobacterium are partially species dependent. Here we characterize the species and subspecies of Bifidobacterium present in breastfed infants around the world. Across populations, three distinct patterns of Bifidobacterium colonization emerged: 1) Dominance of Bifidobacterium longum subspecies infantis, 2) Prevalent Bifidobacterium of multiple species, and 3) Frequent absence of Bifidobacterium. These patterns appear related to country history of breastfeeding, with infants in countries with historically high rates of long duration breastfeeding more likely to be colonized by B. longum subspecies infantis compared with infants in countries with histories of shorter duration breastfeeding. These findings highlight the need to consider historical and cultural influences on gut commensal survival influence present day colonization patterns in order to understand epidemiological transmission patterns of Bifidobacterium and other major gut commensals.
\end{abstract}


Keywords: infant; breastfeeding; gut microbiome; Bifidobacterium

\section{Introduction}

Diet is a major driver of the infant gut microbiome. ${ }^{1}$ The earliest studies of the infant gut microbiome were conducted a century ago and highlight the difference in the gut microbiome of breastfed infants and formula fed infants, with breastfed infants exhibiting near monocultures of Bifidobacterium and formula fed infants exhibiting a more mixed microbiome without Bifidobacterium. ${ }^{2}$ The ability of Bifidobacterium to dominate the breast-fed infant gut is driven, in part, by human milk oligosaccharides (HMOs), ${ }^{3-5}$ the third most abundant solid component of human milk.6,7 Despite the abundance of HMOs in human milk, these specialized carbohydrates are not digested by the infant. ${ }^{7}$ Instead, HMOs act as prebiotics for beneficial bacteria, including Bifidobacterium, and as decoy receptors for pathogens. ${ }^{8}$ Some Bifidobacterium are efficient consumers of HMOs, to the point that HMOs are identified as the "bifidogenic factor" of human milk.3,9

Not all Bifidobacterium are equally efficient at consuming HMOs, ${ }^{10}$ and different species of Bifidobacterium can have different implications for infant health. ${ }^{11}$ Bifidobacterium is a genus composed of 54 different species that had been identified as of January 2017.12 Some Bifidobacterium, such as B. longum subsp. infantis, are efficient consumers of HMOs, while others, such as $B$. longum subsp. longum are better adapted for consumption of plant oligosaccharides. ${ }^{10}$ Bifidobacterium colonization has a number of potential health benefits for infants, including reduction of allergies, ${ }^{13,14}$ improved vaccine response, ${ }^{15}$ reduced carriage of antimicrobial resistance genes, ${ }^{16}$ reduced carriage of virulence factor genes, ${ }^{17}$ reduced enteric inflammation, ${ }^{18}$ and immunoregulation via microbial metabolites. ${ }^{19}$ However, some of these health benefits are dependent on which species of Bifidobacterium are present in the gut microbiome. ${ }^{15,20}$ Therefore, it is important to understand variation in patterns of colonization with Bifidobacterium in infant populations around the world.

Recent work demonstrates the importance of microbial-accessible carbohydrates (MAC) to maintaining commensal species in host microbiomes. ${ }^{21}$ MACs are carbohydrates that are available for fermentation by the microbiome but are not digested by the host. Mouse work has shown that to understand microbes in the present, past dietary practices must also be considered, as populations of mice switched between low and high MAC diets experienced extinctions of portions of the microbes previously present. ${ }^{21}$ For breastfed infants, human milk oligosaccharides (HMOs) constitute a major component of milk and function as MACs. Human milk substitutes have typically lacked HMOs, although a few formulas first introduced in 2018 do contain a single HMO. Despite this, HMOs are not typically included in infant formula, and when they are included, the diversity of HMO structures is far below that found in human milk. As a result, infants fed artificial formulas are consuming a diet low in HMOs, and therefore a diet that is low MAC. Because loss of MACs in the diet can drive extinctions in the gut microbiome, ${ }^{21}$ populations that experienced periods of low breastfeeding and high formula feeding may have lost species of Bifidobacterium most dependent on HMO consumption. In fact, there is already some evidence that this has occurred in 
Western populations as B. longum subsp. infantis has become increasingly difficult to detect in these populations, even in infants never exposed to antibiotics. ${ }^{22}$ Therefore, historical breastfeeding patterns must be considered in addition to current breastfeeding in order to understand the relationship between breastfeeding and the infant gut microbiome in the present day. Infants from populations that experienced periods of lower breastfeeding initiation and shorter duration may be living in regions where HMO-consuming Bifidobacterium are near extinction due to loss in prior generations.

In particular, we hypothesize that commensals such as B. longum subsp. infantis dependent on HMO consumption may be lost from populations with a history of lower breastfeeding initiation and duration while Bifidobacterium species capable of consuming both HMOs and plant oligosaccharides may have a better chance at surviving in populations that have experienced low breastfeeding rates. Here we work to describe the species of Bifidobacterium found in global infant populations, and to consider how population history of breastfeeding practices relates to the currently observed patterns.

\section{Methods}

\section{Inclusion Criteria for Cohorts}

For this analysis, published and unpublished cohorts of term infants where at least some infants were breastfed were selected for inclusion. Cohorts needed to have a minimum of 20 infants and have species-level data on the relative abundance of the Bifidobacterium as measured by $16 S$ rRNA gene sequencing and Bifidobacterium specific terminal restriction fragment length polymorphism (Bif-TRFLP) and Bifidobacterium Longum-Infantis Ratio (BLIR) analysis in infant stool between ages 1 or 2 months, or have 16S rRNA gene sequencing data available with DNA available for species level analysis of Bifidobacterium. Publicly available datasets were included from Gambia, ${ }^{23}$ Bangladesh, ${ }^{15}$ and the PASTURE cohorts from Austria, Finland, Germany, and Switzerland, ${ }^{24,25}$ although this is the first publication to include species level Bifidobacterium data from the PASTURE cohorts. Additionally, data from a subset of infants enrolled in the University of California Davis Lactation Study (Davis, California) 26,27 $^{2}$ and a subset of infants enrolled in the Pediatric Respiratory and Enteric Virus Acquisition and Immunogenesis Longitudinal (PREVAIL) study (Cincinnati, $\mathrm{OH})^{28}$ were included. Table 1 summarizes the cohorts, and lists the total number of infants in each cohort, the number of infants who had a sample from ages 1 to 2 months for inclusion in this study, and the number of infants who were at least partially breastfed at time of sample collection. Historically breastfeeding patterns were defined by searching the literature for references on breastfeeding rates published between 1900 and the present day. A high breastfeeding pattern was defined as one where breastfeeding initiation was nearly universal and breastfeeding duration was typically longer than 1 year. The medium breastfeeding pattern was defined as high current and past rates of breastfeeding initiation, but with evidence of historically short (median less than 6 months) duration. The low breastfeeding pattern was defined as occurring in the case of a documented period where at least half of infants were never breastfed (meaning breastfeeding was not initiated), regardless of current population breastfeeding initiation and duration. While breastfeeding rates for each cohort are calculated using all infants with available breastfeeding data in each cohort 
(Table 1), only infants who were at least partially breastfed at the time of the month 1 or 2 sample are included in all subsequent analyses in this paper. The UC Davis Lactation Study was approved by the UC Davis Institutional Review Board, and all mothers provided written informed consent for their and their infant's participation in this study. The PREVAIL cohort was approved by the Centers for Disease Control and Prevention (CDC) Institutional Review Board and the Cincinnati Children's Hospital Medical Center Institutional Review Board, and all mothers provided written informed consent for their and their infant's participation in this study. For the PASTURE cohorts, the study was approved by local research ethics committees and written informed consent was obtained from the infant's parents. The Bangladeshi cohort was approved by Research Review Committee (RRC) and Ethical Review Committee (ERC) of International Centre for Diarrhoeal Disease Research, Bangladesh (icddr,b). WHO Ethics Review Committee also approved the protocol. Mothers provided written informed consent. For the Gambian cohort, all data were used for this project were publicly available. ${ }^{23}$

For each cohort, data on median duration and initiation rates of breastfeeding are reported if available for the cohort. In addition, historical breastfeeding practices of each country of origin of a cohort are described based on reviews of the published literature. In addition to the month 1-2 samples described above, six cohorts had available longitudinal samples. The Bangladesh cohort had additional samples from a subset of infants at age 2 years with 16S rRNA gene sequencing and Bif-TRFLP/BLIR. The four PASTURE cohorts had additional samples from age 1 year on a subset of infants. The Davis cohort had additional samples from a subset of infants at 3 days of life and age 1 month with 16S rRNA gene sequencing and Bif-TRFLP/BLIR analysis. These cohorts will permit a limited examination of Bifidobacterium colonization over time.

\section{S rRNA Gene Sequencing and Bif-TRFLP/BLIR}

All infant stool samples were extracted and sequenced as described in Davis et al. ${ }^{23}$ Analysis of the $16 \mathrm{~S}$ rRNA gene sequencing results of all raw data files was completed using QIIME229 (version qiime2-2017.8) and DADA2.30 Identification of Bifidobacterium species and B. longum subsp. was completed using Bifidobacterium specific terminal restriction fragment length polymorphism (Bif-TRFLP) and Bifidobacterium Longum-Infantis Ratio (BLIR) as described in Davis et al. ${ }^{23,31}$ Bif-TRFLP and BLIR are well validated by culture for the identification of Bifidobacterium species and subspecies, ${ }^{23,31}$ and enable efficient and cost-effective identification of Bifidobacterium in a large number of samples.

Relative abundance of total Bifidobacterium was compared by cohort and historical breastfeeding pattern using a Kruskal-Wallis test, followed by Dunn's test with Bonferroni correction if results were significant. Prevalence of Bifidobacterium species present in at least two cohorts were compared based on breastfeeding history in each cohort using generalized estimating equations (GEE) as implemented in the gee package in $\mathrm{R}$ version 3.6.3 with a binomial family and logit linker, cohort of origin used as the clustering variable, and using an exchangeable correlation structure. A Bifidobacterium species was considered present in an infant if there was any detectable level of that species in the infant's microbiome. A Bifidobacterium species was 
considered present in a cohort if that species was present in at least one infant in that cohort. The species included for this analysis were B. adolescentis, $B$. animalis, B. bifidum, B. breve, B. longum subsp. infantis, B. longum subsp. longum, B. longum of unknown subsp., and B. pseudocatenulatum. This meant eight separate models were constructed, leading to a Bonferroni corrected pvalue of less than 0.0062 to be considered significant after adjusting for multiple comparisons.

To understand the extent to which individual species of Bifidobacterium shape the microbiome, we sought to identify species that, when present in an infant, typically dominated ( $>50 \%$ median relative abundance) or that typically contributed a substantial fraction towards dominance $(>20 \%$ median relative abundance) in each cohort. To do this, for each species of Bifidobacterium in each cohort we selected only infants who were colonized by detectable levels of that species, then calculated the median relative abundance in the colonized infants.

Finally, in the cohorts with samples from infants collected at older ages, Chi-square tests were used to compare the chances of detecting B. longum subsp. infantis in infants who were still breastfed compared to those who were weaned.

\section{Results}

Study cohorts and breastfeeding patterns.

This study included eight cohorts with a total of 979 breastfed infants with stool samples collected at age 1 or 2 months. Two cohorts were conducted in two low income countries - Bangladesh and the Gambia - and six cohorts were conducted in five high income countries - Austria, Finland, Germany, Switzerland and the United States (US). The countries included have three historically different breastfeeding patterns, described here as "high", "medium", and "low" breastfeeding.

The first breastfeeding pattern, "high", is characterized by consistent, high rates of breastfeeding initiation and long duration of breastfeeding without evidence of historical interruptions in either breastfeeding initiation or duration. This is the pattern observed in The Gambia and Bangladesh. In the 1980s, when breastfeeding was low in much of the world, 97.5\% of Bangladeshi children were fed at least some breastmilk and the mean duration of breastfeeding was 26 months ${ }^{32}$. Furthermore, this pattern of high rates of breastfeeding and long duration remained consistent in studies of Bangladesh from the 1970s through the 1990s. ${ }^{33}$ More recently, the initiation of breastfeeding in Bangladesh remains high (98.3\%) and duration of breastfeeding has increased to a mean of 31.9 months. ${ }^{34}$ The Gambia has a similar historical and modern pattern, where a report from 1979 points out that "all Gambian women breast feed their babies for up to two years." 35 Despite a shorter than optimal duration of exclusive breastfeeding, breastfeeding rates remain high in the Gambia today, with a median duration of 20 months with over $95 \%$ of infants ever breastfed. ${ }^{36}$ Therefore, in this study, infants in the Bangladesh and Gambian cohorts were therefore considered to have a "high" historical breastfeeding pattern. Median duration of breastfeeding is unknown for the Gambian cohort, but all infants in this group were breastfed for at least 5 months as inclusion criteria included the availability of week 20 milk samples. ${ }^{23}$ Median duration of breastfeeding in the Bangladeshi cohort 
is also unknown as infants in this cohort were only followed until age 2 years, and more than half of the infants enrolled in the study were still breastfed at this time point (Table 2). This means that the high breastfeeding pattern is still observed in the present day in these countries.

The second breastfeeding pattern, "medium", is characterized by high, consistent breastfeeding initiation but historical evidence of short duration of breastfeeding (less than 6 months of any breastfeeding in the majority of infants). This pattern is observed in Austria, Finland, Germany, and Switzerland, with historical documentation of a median duration of breastfeeding of less than 6 months during the 1970s and 1980s. In European countries, there was a general decline in breastfeeding following World War II followed by increased rates of breastfeeding starting in the 1970s. ${ }^{37}$ In Austria, Germany, and Finland, in the 1970s and 1980s most women initiated breastfeeding, but duration was short. ${ }^{38}$ In Austria, only 5\% of infants were breastfed at 3 months post-partum in 1980, which increased to $41 \%$ by $1984,{ }^{39}$ indicating that the majority of infants did not receive a full six months of breastfeeding during the early 1980s. In Finland in the early 1980s, most women initiated breastfeeding but only a third of infants were breastfed until 3 months, ${ }^{40}$ again indicating a historical period where the majority of infants were not at least partially breastfed for six months. In Germany, only $2 \%$ of infants were still breastfed at age 6 months in 1980. ${ }^{41}$ In Switzerland, in 1978, 92\% of mothers initiated breastfeeding, however only $30 \%$ were still breastfeeding by 4 months. ${ }^{42}$ These past patterns are no longer present in these countries. In Austria today, breastfeeding initiation rates are currently about $98 \%$ with a median duration of 27 weeks. ${ }^{43}$ In Finland today, almost all mothers initiate breastfeeding, $60 \%$ are still breastfeeding at 6 months and a third are still breastfed at 11 months of age. ${ }^{44}$ In Germany, $90 \%$ of women initiate breastfeeding and more than half of all infants are still breastfed at 6 months. ${ }^{45}$ In Switzerland, breastfeeding initiation is $95 \%$ and the median duration of any breastfeeding is 31 weeks. ${ }^{46}$ These numbers are consistent with those observed for the study cohorts (Table 2). Therefore, even though present day practices in these countries would qualify for the "high" category, historical evidence supports a period with low HMO consumption by infants creating the opportunity for shifts in infant Bifidobacterium populations.

The third pattern of breastfeeding, "low", is characterized by low historic rates of breastfeeding initiation. The two cohorts from the United States meet this pattern, as the United States experienced a low point in breastfeeding in 1971 when less than 1 in 4 women breastfed their infants even once. ${ }^{47}$ Following sustained public health efforts, rates partially recovered to approximately $50 \%-60 \%$ initiating breastfeeding in the 1980 s. ${ }^{48}$ Breastfeeding initiation remained at only $60 \%$ in 1995 , with only $22 \%$ of mothers still breastfeeding at age 6 months in $1995 .{ }^{49}$ As recently as 2004, the United States had lower breastfeeding initiation and duration rates than many continental European countries. ${ }^{50}$ Since that time, breastfeeding in the US has increased so that in $2017,84 \%$ of infants were ever breastfed and $58 \%$ were still breastfed at 6 months. ${ }^{51}$ In the state of Ohio (where Cincinnati is located) in 2017 (the year that the Cincinnati PREVAIL cohort began enrollment), $80 \%$ of infants were ever breastfed and $51 \%$ were still breastfed at age 6 months. ${ }^{51}$ For the state of California (where Davis is located) in 2009 (the year the UC Davis Lactation Cohort began enrollment), $85 \%$ of infants were ever breastfed and $53 \%$ were 
still breastfed at age 6 months. ${ }^{52}$ Because the Davis Lactation Cohort enrolled based on the intention to breastfed, this cohort has a longer median duration of breastfeeding than was observed in the general population (Table 2). The Cincinnati cohort had a lower duration of breastfeeding (Table 2) than that reported for Ohio, in part because this cohort is an urban, diverse cohort which tends to mean a shorter duration of breastfeeding.

Bifidobacterium species across Cohorts and Breastfeeding patterns.

Average Bifidobacterium levels differed between cohorts (Figure 1). The prevalence of detection of any Bifidobacterium was $100 \%$ of infants in all cohorts except those in the United States. The total relative abundance of Bifidobacterium in individual infants also differed significantly by cohort (Kruskal-Wallis test, $\mathrm{p}<0.0001$ ) and by breastfeeding pattern (Kruskal-Wallis test, $\mathrm{p}<0.0001)$. By cohort, Davis had significantly lower total relative abundance of Bifidobacterium than all other cohorts (Dunn's test, $\mathrm{p}<0.0001$ compared to all cohorts except Cincinnati where $\mathrm{p}=0.03$ ). Switzerland had significantly higher relative abundance of Bifidobacterium than all other cohorts except for Gambia (Dunn's test, $\mathrm{p}<0.0001$ for all cohorts except Gambia, no significant difference in total Bifidobacterium between Switzerland and Gambia, $\mathrm{p}=1.0$ ). There were no other significant differences in total Bifidobacterium relative abundance by cohort. By historical breastfeeding status, the low breastfeeding pattern infants had a lower relative abundance of Bifidobacterium than the high breastfeeding pattern infants (Dunn's test, $\mathrm{p}<0.0001$ ) and the medium breastfeeding pattern infants (Dunn's test, $\mathrm{p}<0.0001$ ). The medium breastfeeding pattern infants had higher total Bifidobacterium than the high breastfeeding infants (Dunn's test, $\mathrm{p}=0.02$ ).

Importantly, the cohorts differed in the species of Bifidobacterium that colonized infant guts. The average infant gut microbiome in Bangladesh and Gambia is dominated (meaning greater than 50\% relative abundance Bifidobacterium of a single subspecies) by B. longum subsp. infantis, with an average relative abundance of $54 \%$ B. longum subsp. infantis in Bangladesh and $53 \%$ in the Gambia. In the European and USA cohorts, the average infant gut microbiome was not dominated by any single species of Bifidobacterium (Figure 1).

The presence or absence of particular Bifidobacterium species in infants reflected historical breastfeeding practices among the cohorts (see Table 3 for the summary of findings from GEE models). Notably, B. longum subsp. infantis and B. bifidum were significantly less likely to be present in infants from medium and low breastfeeding pattern cohorts compared to infants in high breastfeeding pattern cohorts. B. pseudocatenulatum was more likely to be present in infants from medium or low breastfeeding pattern cohorts than in infants from high breastfeeding pattern cohorts. B. adolescentis and B. breve were detected more often in medium breastfeeding pattern cohorts than in high breastfeeding pattern cohorts, but there was no significant difference in the presence of these species between low breastfeeding pattern cohorts and high breastfeeding pattern cohorts. The prevalence of these species in each cohort is shown in Table 2. Because B. breve, B. pseudocatenulatum, and B. bifidum may be difficult to distinguish when using Bif-TRFLP, the values in Table 2 may underestimate the true prevalence of these taxa in all cohorts. All figures include the relative abundance of these mixed peaks as "Unknown 
Bifidobacterium", but indistinguishable peaks do not count towards the estimates of relative abundance for these species or towards the presence or absence of these species in any infant.

Detailed visualizations of Bifidobacterium colonization of individual infants in each cohort demonstrate the substantial concordance between historical breastfeeding patterns and infant gut colonization (Figure 2). The similarities of infant Bifidobacterium colonization patterns by historical breastfeeding are striking. A majority of infants in the high historical breastfeeding cohorts having gut microbiomes dominated by B. longum subsp. infantis with lower relative abundance of other Bifidobacterium species (Figure 2A and 2B). In the medium historical breastfeeding cohorts, infants still have very high levels of total Bifidobacterium, but no single species dominates (Figure 2C-2F). In the low historical breastfeeding cohorts, some breastfed infants completely lack Bifidobacterium, but those who are colonized by Bifidobacterium generally appear similar to the pattern observed in the medium historical breastfeeding cohorts. As medium breastfeeding pattern infants had higher total Bifidobacterium levels than high breastfeeding pattern infants and because there were significant differences in the presence and absence of specific Bifidobacterium species, we examined which Bifidobacterium could dominate the infant gut microbiome. In the high historical breastfeeding pattern cohorts, approximately two-thirds of infants had a gut microbiome dominated (meaning greater than $50 \%$ relative abundance from a single source) by B. longum subsp. infantis. In addition, B. breve, B. longum subsp. longum, and B. pseudocatenulatum or a mixture of multiple Bifidobacterium species would occasionally result in an infant gut microbiome dominated by Bifidobacterium. In the medium historical breastfeeding pattern cohorts, a microbiome dominated by $B$. longum subsp. infantis was rare, but roughly two-thirds of infants had a gut microbiome dominated by B. breve, B. longum subsp. longum, and B. pseudocatenulatum or a mixture of multiple Bifidobacterium species. In the low historical breastfeeding pattern cohorts, some breastfed infants completely lacked any detectable level of Bifidobacterium. When infants did have gut microbiomes dominated by Bifidobacterium, it was typically the same mix of species as observed in the medium historical breastfeeding pattern cohorts. On occasion, B. bifidum could also dominate an infant gut microbiome, but this was a rare occurrence with only 1 infant in the German cohort, 1 infant in the Finnish cohort, and 1 infant in the Davis, USA cohort.

\section{Timing and Bifidobacterium Species Colonization}

Six cohorts, the Bangladesh cohort, the four PASTURE cohorts (from Austria, Finland, Germany, and Switzerland), and the UC Davis lactation cohort, had additional longitudinal data on infant Bifidobacterium colonization patterns, although the timing of the available data differed. We analyzed these additional time points to address questions of timing regarding the acquisition of Bifidobacterium species (in the UC Davis cohort) and the loss or consistency of colonization with Bifidobacterium species comparing early infancy to 1 or 2 years of age (Bangladesh cohort and PASTURE cohorts).

There were differences over time in the colonization of Davis infants with B. longum subsp. infantis (Figure 3). Figure 3A again shows the 2 month Bifidobacterium colonization of the Davis infants, the stars indicate the infants colonized by $B$. infantis who also had samples at an earlier time point. 
Regarding timing of acquisition of Bifidobacterium species, the UC Davis lactation cohort had additional samples with known Bifidobacterium colonization patterns from day 3 of life (29 infants; Figure 3B) and month 1 of life (30 infants; Figure 3C) in addition to the month 2 samples described above. The median relative abundance of total Bifidobacterium was only $1 \%$ at day 3 (range $0 \%-91 \%$ ), with $38 \%$ of infants having no detectable level of Bifidobacterium present. The species of Bifidobacterium detected at day of life 3 were $B$. longum subsp. longum (detected in $28 \%$ of infants), B. bifidum (in $14 \%$ of infants), B. breve (in 14\% of infants), B. pseudocatenulatum (in 14\% of infants), and $B$. adolescentis (in 3\% of infants) (Figure $4 \mathrm{~A}$ ). At age one month, 30 of the infants (including all 29 with day of life 3 samples) had additional BifTRFLP/BLIR results (Figure $4 \mathrm{~B}$ ). $43 \%$ of Davis infants had no detectable Bifidobacterium at month 1 of life, and a median relative abundance of total Bifidobacterium of $0.2 \%$ (range $0 \%-95 \%$ ). The species that were present were B. longum subsp. longum (detected in $23 \%$ of infants), B. bifidum (in $23 \%$ of infants), B. breve (in 23\% of infants), B. adolescentis (in 10\% of infants), and $B$. pseudocatenulatum (in $7 \%$ of infants). Notably, B. longum subsp. infantis was not found in any Davis infants prior to age 2 months.

One hundred and nine of the 274 Bangladeshi infants had data on Bifidobacterium colonization at age 2 years. At age 2, all of these infants were still colonized with at least some Bifidobacterium, but the median total relative abundance of Bifidobacterium was lower than in early life at $21 \%$ relative abundance Bifidobacterium (range 3\% - 82\%; Figure 4). Furthermore, the prevalence of $B$. longum subsp. infantis had dropped considerably and was found in just $17 \%$ of infants overall, including $20 \%$ of still breastfed infants and $11 \%$ of infants who had not received breastmilk in the past week (Table 4 ). In contrast to the dominance of B. longum subsp. infantis in early infancy, the species of Bifidobacterium found in the Bangladeshi infant at age 2 years, in order of decreasing prevalence, were B. pseudocatenulatum (detected in $95 \%$ of infants), B. longum subsp. longum (detected in $95 \%$ of infants), B. bifidum (detected in $78 \%$ of infants), B. breve (detected in $65 \%$ of infants), B. adolescentis (detected in $32 \%$ of infants), B. longum subsp. infantis (detected in $17 \%$ of infants), $B$. animalis (detected in $9 \%$ of infants), B. magnum (detected in $2 \%$ of infants), and an indistinguishable mix of B. choerinum and/or B. pseudolongum ( $1 \%$ of infants, this indistinguishable mix was also plotted as part "Unknown Bifidobacterium").

In the Austrian cohort, 91 of the infants breastfed at 2 months also had a year 1 sample. In the Finnish cohort, 129 of the infants breastfed at 2 months also had a year 1 sample. In the German cohort, 110 of the infants breastfed at 2 months also had a 1 year sample. In the Swiss cohort, 175 of the infants breastfed at 2 months also had 1 year sample. All infants were still colonized with at least some Bifidobacterium (Figure 5), but usually at a lower relative abundance of total Bifidobacterium than was present at 2 months of age. Comparing colonization with $B$. longum subsp. infantis between infants who were or were not still breastfed at age 1 year, infants who were breastfed always had a higher prevalence of B. longum subsp. infantis than those who were not, but this difference only reached significance in the Austrian (Chi-square test, $\mathrm{p}=0.003$ ) and Swiss (Chi-square-test, $\mathrm{p}<0.001$ ) cohorts (Table 4). The greater prevalence of $B$. longum subsp. infantis in breastfed at 1 year infants compared to not breastfed at 1 year infants in the Austrian and Swiss cohorts is 
consistent with horizontal transmission of B. longum subsp. infantis, as this species does not remain at high rates in infants who are no longer breastfed. Furthermore, across all the European cohorts, there were 28 infants with detectable levels of $B$. longum subsp. infantis at month 2 but not at year 1, 18 infants with detectable levels of $B$. longum subsp. infantis at year 1 but not at month 2, and 9 infants with detectable levels at both time points. Of the 18 infants who acquired B. longum subsp. infantis between 2 months and 1 year, $13(72 \%)$ were breastfed at 1 year of age while only $5(28 \%)$ were not breastfed at 1 year of age. In contrast, of the 28 infants who lost $B$. longum subsp. infantis between month 2 and year 1, 26 (93\%) were not breastfed at age 1 year and only $2(7 \%)$ were breastfed at age 1 year. This is consistent with the idea that infants who are breastfed are more likely to acquire B. longum subsp. infantis and that this subspecies is likely to be lost after the end of breastfeeding. The mix of Bifidobacterium species found in these infants at 1 year of age was generally similar to those observed at 2 months (Table 5).

\section{Discussion}

In our study of cohorts from across the world, we found that the infants from high historical breastfeeding pattern countries are more likely to be colonized with B. longum subsp. infantis and B. bifidum than infants from other cohorts. The pattern of Bifidobacterium colonization by cohort is consistent with the apparent loss of $B$. longum subsp. infantis in locations of the world with historically lower breastfeeding rates and shorter breastfeeding durations. In individual infants, several Bifidobacterium can at least occasionally dominate the gut microbiome. However, in this study only B. longum subspecies infantis dominated the gut microbiome in more than half of the infants where it is highly prevalent. In the relative absence of B. longum subsp. infantis, the infants from medium and low breastfeeding pattern countries, three species are likely to contribute substantially to the dominance of total Bifidobacterium including B. breve, B. longum subsp. longum, and B. pseudocatenulatum. Two of these species, B. longum subsp. longum and B. pseudocatenulatum, are also more likely to colonize infants from medium and low breastfeeding countries. This suggests that these species may contribute more to the infant gut microbiome when species tied tightly to HMO consumption are absent. Infants from medium breastfeeding pattern countries also had higher rates of colonization with $B$. adolescentis and $B$. breve than high breastfeeding pattern countries. This loss of B. longum subsp. infantis in medium and low breastfeeding countries opens an ecological niche for other Bifidobacterium, creating a situation where infants from medium breastfeeding countries frequently have the same relative abundance of Bifidobacterium as infants from high breastfeeding pattern countries, but the Bifidobacterium are from multiple species rather than dominated by just the single subspecies as occurs with $B$. longum subsp. infantis. The infants from low breastfeeding pattern cohorts sometimes lack detectable Bifidobacterium, but otherwise appear similar to the infants of medium breastfeeding cohorts. Despite its rarity, when B. longum subsp. infantis is detected in infants from the medium and low breastfeeding pattern cohorts, it typically dominates the infant gut microbiome of those infants similar to the pattern observed in the high breastfeeding country infants. 
The timing of colonization with B. longum subsp. infantis is distinct from the timing of colonization that occurs with other Bifidobacterium. Most species of Bifidobacterium appeared early in the Davis cohort, suggesting a pattern of vertical transmission consistent with prior work on the transmission of Bifidobacterium. ${ }^{53}$ B. longum subsp. Infantis, however, did not appear as a part of any infant's gut microbiome until age 2 months, suggesting that this particular subspecies may not transmit vertically from the mother. The longitudinal data from the Bangladeshi cohort further supports our hypothesis, as B. longum subsp. infantis is found in $84 \%$ of infants at age 6 weeks, but only $17 \%$ of infants at age 2 years. This suggests that even in populations where $B$. longum subsp. infantis is commonly found in infants, this subspecies is unlikely to persist in adults as it is replaced by a mix of species similar to those seen in infants from other populations as breastmilk becomes a smaller portion of the diet and the gut microbiome matures. As only $17 \%$ of 2 year olds are colonized by B. longum subsp. infantis it is unlikely that $84 \%$ of women are colonized with B. longum subsp. infantis to transmit it vertically. This is also consistent with a horizontal transmission of $B$. longum subsp. infantis between infants. The results from the European cohort also support this trend, as the infants who are breastfed are more likely to be colonized by B. longum subsp. infantis than those who are not, and a number of infants who did not have detectable levels of $B$. longum subsp. infantis at 2 months of age had detectable levels at age 1 year if they remained breastfed.

The pattern of increased prevalence of B. longum subsp. infantis in cohorts with an uninterrupted history of long duration breastfeeding is consistent with other studies. Consider a population in the US that never widely adopted formula: Old Order Mennonites. Old Order Mennonites are unusual in the United States because they have never widely adopted formula use, and generally avoid antibiotic use. ${ }^{44}$ Consistent with what is discussed in the cohorts included in this study, colonization by B. longum subsp. infantis or with any Bifidobacterium is more prevalent in the Old Order Mennonites, with B. longum subsp. infantis detected by qPCR in 70\% of the Old Order Mennonite infants. ${ }^{13}$ As $B$. longum subsp. infantis is genetically equipped to consume HMOs ${ }^{10}$ and formula lacks HMOs, this supports the concept of microbial accessible carbohydrates (MACs) or their lack driving extinctions of microbial commensals within populations. The low MAC diet associated with historical formula use may explain the disappearance of B. longum subsp. infantis from European and United States cohorts. The higher breastfeeding initiation rates in the European countries may have permitted some species of Bifidobacterium to continue to circulate at higher levels in infants than what was possible in the US, particularly if the species in question are also found in the guts of adults and older children. Many adult strains of Bifidobacterium have at least some of the genetic machinery needed to consume HMOs ${ }^{10}$ and may therefore move in to take advantage of a niche left open by the missing B. longum subsp. infantis.

This study does have limitations, including the small number of cohorts included. Importantly, the limited number of cohorts is due, in part, to selecting only cohorts of infants with species level identification of Bifidobacterium completed using the same technique during a narrow infant age window. This careful limitation to a single method permits direct comparisons across cohorts that would not be possible if a broader range of time points or 
methods were included. The small number of included cohorts is balanced by the more than 900 breastfed infants included in this study. An additional strength of this study is the inclusion of cohorts from four different continents and a clear gradient in breast-feeding history across cohorts. Using BifTRFLP/BLIR also presents a challenge, where in some cases species identification may be ambiguous. This imprecision in species identification is particularly common with B. bifidum, B. breve, and B. pseudocatenulatum as these three species produce peaks of very similar size. Most of the unknown Bifidobacterium reported in the plots is related to ambiguous peaks generated by some strains of B. bifidum, B. breve, and B. pseudocatenulatum. This suggests that abundance and prevalence estimates for these three species may be lower than what is reported here. B. bifidum occurred more frequently in Bangladesh than in any other cohort, but was completely absent in the Gambia. As the number of infants in the Gambian cohort was small, this may represent a lower prevalence of $B$. bifidum, perhaps similar to what is seen in the European countries rather than true absence. Another possibility is that $B$. bifidum is present in this cohort, but undetected because of the similarity of $B$. bifidum to B. pseudocatenulatum and B. breve when using Bif-TRFLP. Because of these limits in the data, it is difficult to draw conclusions about B. bifidum global colonization patterns from this work. The limitation in separating out B. bifidum, B. breve, and B. pseudocatenulatum is counter-balanced by the improved ability to distinguish $B$. longum subsp. infantis from B. longum subsp. longum, even in the event that both subspecies are found in the same infant with one at much lower levels than the other. As B. longum subsp. infantis has been proposed to be endangered in western countries, ${ }^{55}$ a method that could reliably and efficiently distinguish these two subspecies was critical to this work on how breastfeeding fits into the concept of microbial accessible carbohydrates. An additional limitation of this work is the narrow focus on breastfeeding; there are several additional factors not considered in this work that may be important for Bifidobacterium colonization patterns. These factors include prevalence of antibiotic use in the different cohorts, infant care patterns (e.g. when infants are sent to out of home childcare ${ }^{56}$ ), and hygiene practices. These metadata were not consistently collected for the included cohorts so they could not be considered in the present study. Future work is needed to understand the complex set of factors that support Bifidobacterium transmission both historically and in the present day. For the present study, we were less concerned about antibiotic use than diet, because antibiotic use is prevalent in Bangladesh, where the prevalence of B. longum subsp. infantis remains high. For instance, approximately $40 \%$ of children under the age of 5 with an acute respiratory infection in Bangladesh receive antibiotics. ${ }^{57}$ This suggests that prevalent antibiotic use is less likely to be a driving factor in the missing B. longum subsp. infantis than diet.

Bifidobacterium are important infant commensals, but the full health implications of colonization by different species of Bifidobacterium remains unclear. This is despite evidence that colonization by Bifidobacterium is important to health and evidence that some Bifidobacterium species may be more effective at supporting infant health than others. Some benefits of high levels of Bifidobacterium colonization do not appear to be species dependent, such as reduced carriage of antimicrobial resistance genes. ${ }^{16}$ Other benefits of high levels of Bifidobacterium are species dependent. For example, higher levels of 
B. longum subsp. infantis are associated with improved vaccine response, but the same association is not seen with higher levels of $B$. longum subsp. longum or B. breve. ${ }^{15}$ B. longum subsp. longum is found more often in healthy infants than in those with allergic symptoms, but the same trend was not seen for other Bifidobacterium species. ${ }^{58}$ B. longum subsp. infantis was found more frequently in Old Order Mennonite infants with lower risk of atopic disease than in Rochester infants. ${ }^{13}$ As such, understanding the differences in Bifidobacterium species colonization in infant populations is important when studying the health implications of the early life microbiome. The fact that the health implications of Bifidobacterium vary by species and that Bifidobacterium species colonization patterns vary by country and population history of breastfeeding mean care needs to be taken in interpreting health findings associated with of high levels of Bifidobacterium without specifying species if trying to apply the findings to other populations. This means it is critical to understand the epidemiological factors that support transmission of different species of Bifidobacterium, including considering how historic formula-driven extinctions may have changed the colonization patterns observed in the present day.

Author Contributions: DHT, ZTL, NN, SH, CM, VDC, MNH, CBS, EvM, PK, JCD, RL, JR, JTS, JBG, ALM, and DAM all contributed to the acquisition of data. DHT, ZTL, DJT, ALM, and DAM contributed to data analysis. DHT, ZTL, KH, ALM, and DAM contributed to the conception and design of this study. DHT, ZTL, ALM, and DAM drafted the initial manuscript. All authors critically reviewed and approved the final manuscript, except JCD who passed away before the manuscript draft was complete.

Funding: Funding for this project includes US Department of Agriculture - Agricultural Research Service project 5306-51530-018-00, World Health Organization project 2010168947, Thrasher Research fund grant 11488. Funding for this project also includes National Institutes of Health award F32HD093185 (D.H.T.) and the Peter J. Shields Endowed Chair in Dairy Food Science (D.A.M.).

Informed Consent Statement: The Bangladesh, Gambian, and PASTURE cohorts include already published data. The UC Davis Lactation Study was approved by the UC Davis Institutional Review Board, and all mothers provided written informed consent for their and their infant's participation in this study. The PREVAIL cohort was approved by the Centers for Disease Control and Prevention (CDC) Institutional Review Board and the Cincinnati Children's Hospital Medical Center Institutional Review Board, and all mothers provided written informed consent for their and their infant's participation in this study.

Impact Statement: Population history of breastfeeding influences the present-day infant microbiome. Bifidobacterium longum subspecies infantis, an efficient consumer of HMOs, is found most commonly in populations with uninterrupted high rates of breastfeeding initiation and uninterrupted long duration of breastfeeding. Other species of Bifidobacterium are more commonly found in populations with more disrupted breastfeeding histories.

Acknowledgments: Thank you to all the mother-infant dyads who participated in this research. Funding for this project includes US Department of Agriculture - Agricultural Research Service project 5306-51530-018-00, World Health Organization project 2010168947, Thrasher Research fund grant 11488. Funding for this project also includes National Institutes of Health award F32HD093185 (D.H.T.) and the Peter J. Shields Endowed Chair in Dairy Food Science (D.A.M.). 
Conflicts of Interest: David A. Mills and J. Bruce German are co-founders of Evolve Biosystems and BCD Biosciences. Jennifer T. Smilowitz consults with Evolve Biosystems. Evolve Biosystems and BCD Biosciences had no role in the funding, design, data acquisition and analysis, or decision to publish this work. Ardythe L. Morrow is a co-founder of Glycosyn, LLC, which had no role in the funding, design, data acquisition and analysis, or decision to publish this work.

\section{References}

1 Davis, E. C., Wang, M. \& Donovan, S. M. The Role of Early Life Nutrition in the Establishment of Gastrointestinal Microbial Composition and Function. Gut microbes 8, 143-171 (2017).

2 Logan, W. R. The Intestinal Flora of Infants and Young Children. The Journal of Pathology and Bacteriology 18, 527551 (1913).

3 Ward, R. E., Ninonuevo, M., Mills, D. A., Lebrilla, C. B. \& German, J. B. In Vitro Fermentability of Human Milk Oligosaccharides by Several Strains of Bifidobacteria. Molecular nutrition \& food research 51, 1398-1405 (2007).

4 LoCascio, R. G. et al. Glycoprofiling of Bifidobacterial Consumption of Human Milk Oligosaccharides Demonstrates Strain Specific, Preferential Consumption of Small Chain Glycans Secreted in Early Human Lactation. Journal of Agricultural and Food Chemistry 55, 8914-8919 (2007).

5 Sela, D. A. et al. The Genome Sequence of \&Lt;Em\&Gt;Bifidobacterium Longum\&Lt;/Em\&Gt; Subsp. \&Lt;Em\&Gt;Infantis\&Lt;/Em\&Gt; Reveals Adaptations for Milk Utilization within the Infant Microbiome. Proceedings of the National Academy of Sciences 105, 18964 (2008).

6 Newburg, D., Neubauer, S. \& Jensen, R. Handbook of Milk Composition. ed Jensen RG, Academic Press, San Diego, 273-349 (1995).

7 Petherick, A. Development: Mother\&\#39;S Milk: A Rich Opportunity. Nature 468, S5 (2010).

8 Ballard, O. \& Morrow, A. L. Human Milk Composition: Nutrients and Bioactive Factors. Pediatric Clinics 60, 4974 (2013).

9 Bode, L. Human Milk Oligosaccharides: Every Baby Needs a Sugar Mama. Glycobiology 22, 1147-1162 (2012).

10 Sela, D. A. \& Mills, D. A. Nursing Our Microbiota: Molecular Linkages between Bifidobacteria and Milk Oligosaccharides. Trends in microbiology 18, 298-307 (2010).

11 Tannock, G. W. Commentary: Remembrance of Microbes Past. International Journal of Epidemiology 34, 13-15 (2005).

12 Mattarelli, P. \& Biavati, B. in The Bifidobacteria and Related Organisms: Biology, Taxonomy, and Applications (Mattarelli, P., Biavati, B., Holzapfel, W. H. \& wood, B. J. B. eds.) Ch. 2, (Andre G. Wolff, 2018).

13 Seppo, A. E. et al. Infant Gut Microbiome Is Enriched with Bifidobacterium Longum Ssp. Infantis in Old Order Mennonites with Traditional Farming Lifestyle. Allergy n/a (2021).

14 Cukrowska, B., Bierła, J. B., Zakrzewska, M., Klukowski, M. \& Maciorkowska, E. The Relationship between the Infant Gut Microbiota and Allergy. The Role of Bifidobacterium Breve and Prebiotic Oligosaccharides in the Activation of Anti-Allergic Mechanisms in Early Life. Nutrients 12, 946 (2020).

15 Huda, M. N. et al. (Am Assoc Immnol, 2017).

16 Taft, D. H. et al. Bifidobacterial Dominance of the Gut in Early Life and Acquisition of Antimicrobial Resistance. mSphere 3 (2018).

17 Casaburi, G. \& Frese, S. A. Colonization of Breastfed Infants by Bifidobacterium Longum Subsp. Infantis Evc001 Reduces Virulence Gene Abundance. Human Microbiome Journal 9, 7-10 (2018).

18 Henrick, B. M. et al. Colonization by B. Infantis Evc001 Modulates Enteric Inflammation in Exclusively Breastfed Infants. Pediatric Research 86, 749-757 (2019).

19 Henrick, B. M. et al. Bifidobacteria-Mediated Immune System Imprinting Early in Life. Cell 184, 3884-3898.e3811 (2021).

20 He, F. et al. Comparison of Mucosal Adhesion and Species Identification of Bifidobacteria Isolated from Healthy and Allergic Infants. FEMS immunology and medical microbiology 30, $43-47$ (2001).

21 Sonnenburg, E. D. et al. Diet-Induced Extinctions in the Gut Microbiota Compound over Generations. Nature 529, 212-215 (2016).

22 Tannock, G. W., Lee, P. S., Wong, K. H. \& Lawley, B. Why Don't All Infants Have Bifidobacteria in Their Stool? Frontiers in microbiology 7, 834 (2016).

23 Davis, J. C. C. et al. Growth and Morbidity of Gambian Infants Are Influenced by Maternal Milk Oligosaccharides and Infant Gut Microbiota. Scientific Reports 7, 40466 (2017).

24 von Mutius, E. \& Schmid, S. The Pasture Project: Eu Support for the Improvement of Knowledge About Risk Factors and Preventive Factors for Atopy in Europe. Allergy 61, 407-413 (2006). 
25 Depner, M. et al. Maturation of the Gut Microbiome During the First Year of Life Contributes to the Protective Farm Effect on Childhood Asthma. Nature Medicine 26, 1766-1775 (2020).

26 Smilowitz, J. T. et al. The Human Milk Metabolome Reveals Diverse Oligosaccharide Profiles. The Journal of Nutrition 143, 1709-1718 (2013).

27 Lewis, Z. T. et al. Maternal Fucosyltransferase 2 Status Affects the Gut Bifidobacterial Communities of Breastfed Infants. Microbiome 3, 13 (2015).

28 Morrow, A. L. et al. Pediatric Respiratory and Enteric Virus Acquisition and Immunogenesis in Us Mothers and Children Aged 0-2: Prevail Cohort Study. JMIR research protocols 10, e22222 (2021).

29 Caporaso, J. G. et al. Qiime Allows Analysis of High-Throughput Community Sequencing Data. Nature Methods 7 (2010).

30 Callahan, B. J. et al. Dada2: High-Resolution Sample Inference from Illumina Amplicon Data. Nature Methods 13, 581 (2016).

31 Lewis, Z. T. et al. Use of Bifidobacterial Specific Terminal Restriction Fragment Length Polymorphisms to Complement Next Generation Sequence Profiling of Infant Gut Communities. Anaerobe 19, 62-69 (2013).

32 Ferry, B. Breastfeeding (International Statistical Institute

World Fertility Survey, 1981).

33 Greiner, T. Breastfeeding in Bangladesh: A Review of the Literature. Bangladesh J Nutr 10, 37-50 (1997).

34 Akter, S. \& Rahman, M. M. Duration of Breastfeeding and Its Correlates in Bangladesh. Journal of Health, Population, and Nutrition 28, 595-601 (2010).

35 Whitehead, R. Nutritional Requirements: Infant Feeding Practices and the Development of Malnutrition in Rural Gambia. Food and Nutrition Bulletin 1, 1-6 (1979).

36 (GBOS), T. G. B. o. S. (The Gambia Bureau of Statistics (GBOS). 2011).

37 Thorvaldsen, G. Was There a European Breastfeeding Pattern? The History of the Family 13, 283-295 (2008).

38 Kersting, M. et al. [Breast Feeding Studies 1981-1983 in 1,500 Mothers in Dortmund and Haltern. Iii. Rates of Breast Feeding and Duration of Breast Feeding in the First Half Year]. Monatsschrift Kinderheilkunde : Organ der Deutschen Gesellschaft fur Kinderheilkunde 135, 314-319 (1987).

39 Helsing, E. Supporting Breastfeeding: What Governments and Health Workers Can Do - European Experiences. International Journal of Gynecology \& Obstetrics 31, $69-76$ (1990).

40 Verronen, P. Imetys on Muotia (Breast-Feeding Is Popular). Suom Lä̈̈käril 39, 1078-1079 (1984).

41 Brunn, S. in World health forum 1986; 7 (1): 65-68.

42 Tönz, O., Schwaninger, U., Holzherr, E. \& Schafroth, M. [Infant Nutrition in Switzerland 1978. A Prospective Study on the Nutritional Habits During the First 6 Months of Life. I. Natural Nutrition: Breast Feeding]. Schweiz Med Wochenschr 110, 937-947 (1980).

43 Bürger, B. et al. Breastfeeding Prevalence in Austria According to the Who Iycf Indicators-the Sukie-Study. Nutrients 13, 2096 (2021).

44 Hasunen, K. Imeväisikäisten Ruokinta Suomessa Vuonna 2005. (2005).

45 Kohlhuber, M., Rebhan, B., Schwegler, U., Koletzko, B. \& Fromme, H. Breastfeeding Rates and Duration in Germany: A Bavarian Cohort Study. British Journal of Nutrition 99, 1127-1132 (2008).

46 Dratva, J., Gross, K., Späth, A. \& Zemp, E. Swifs-Swiss Infant Feeding Study: A National Study on Infant Feeding and Health in the Child' S First Year: Executive Summary. Basel: Swiss Tropical and Public Health Institute (2014).

47 Wolf, J. H. Low Breastfeeding Rates and Public Health in the United States. American Journal of Public Health 93 , 2000-2010 (2003).

48 Ryan, A. S., Rush, D., Krieger, F. W. \& Lewandowski, G. E. Recent Declines in Breast-Feeding in the United States, 1984 through 1989. Pediatrics 88, 719-727 (1991).

49 Ryan, A. S. The Resurgence of Breastfeeding in the United States. Pediatrics 99, e12-e12 (1997).

50 Callen, J. \& Pinelli, J. Incidence and Duration of Breastfeeding for Term Infants in Canada, United States, Europe, and Australia: A Literature Review. Birth 31, 285-292 (2004).

51 Rupnicki, S. Breastfeeding Report Card United States, 2020. (2020).

52 Health, U. D. o. \& Services, H. Breastfeeding Report Card. National Immunization Survey Available at: http://www cdc gov/breastfeeding/data/report_card2 htm Accessed April 15 (2009).

53 Duranti, S. et al. Maternal Inheritance of Bifidobacterial Communities and Bifidophages in Infants through Vertical Transmission. Microbiome 5, 66 (2017).

54 O'Leary, J. Do Old Order Mennonites Hold the Key to Understanding Food Allergies, $<$ https://www.urmc.rochester.edu/news/story/do-old-order-mennonites-hold-the-key-to-understanding-foodallergies> (2017). 
55 Tannock, G. W. et al. Comparison of the Compositions of the Stool Microbiotas of Infants Fed Goat Milk Formula, Cow Milk-Based Formula, or Breast Milk. Appl Environ Microbiol 79, 3040-3048 (2013).

56 Thompson, A. L., Monteagudo-Mera, A., Cadenas, M. B., Lampl, M. L. \& Azcarate-Peril, M. A. Milk- and SolidFeeding Practices and Daycare Attendance Are Associated with Differences in Bacterial Diversity, Predominant Communities, and Metabolic and Immune Function of the Infant Gut Microbiome. Frontiers in Cellular and Infection Microbiology 5 (2015).

57 Hassan, M. Z. et al. Antibiotic Use for Acute Respiratory Infections among under-5 Children in Bangladesh: A Population-Based Survey. BMJ Global Health 6, e004010 (2021).

58 Akay, H. K. et al. The Relationship between Bifidobacteria and Allergic Asthma and/or Allergic Dermatitis: A Prospective Study of 0-3 Years-Old Children in Turkey. Anaerobe 28, 98-103 (2014). 
Figures and Tables

Table 1: Source of included cohorts, and number of infants included in the present study from each cohort

\begin{tabular}{|c|c|c|c|c|c|c|}
\hline $\begin{array}{l}\text { Country of } \\
\text { Origin }\end{array}$ & Published & Study & $\begin{array}{l}\text { Enrollment } \\
\text { based on intent } \\
\text { to breastfeed? }\end{array}$ & $\begin{array}{l}\text { Total Number } \\
\text { Infants } \\
\text { Cohort }\end{array}$ & $\begin{array}{l}\text { Number of } \\
\text { Infants with } \\
\text { Stool Samples }\end{array}$ & $\begin{array}{l}\text { Number } \\
\text { Breastfed } \\
\text { Infants } \\
\text { of time } \\
\text { collection }\end{array}$ \\
\hline Austria & Partially & PASTURE & No & 207 & 181 & 122 \\
\hline Bangladesh & Yes & \begin{tabular}{|l} 
Efficacy of Newborn \\
Vitamin \\
Supplementation \\
Improving $\quad$ Immune \\
Function \\
(clinicaltrials.gov \\
NTC01583972)
\end{tabular} & No & 306 & 274 & 274 \\
\hline Finland & Partially & PASTURE & No & 171 & 153 & 135 \\
\hline Gambia & Yes & $\begin{array}{l}\text { Sub-study in The Early } \\
\text { Nutrition and Immune } \\
\text { Development (ENID) } \\
\text { Trial, ISRCTN49285450 }\end{array}$ & Yes & 33 & 24 & 24 \\
\hline Germany & Partially & PASTURE & No & 237 & 198 & 149 \\
\hline Switzerland & Partially & PASTURE & No & 231 & 227 & 189 \\
\hline $\begin{array}{l}\text { Davis, } \\
\text { California, } \\
\text { United States }\end{array}$ & Partially & $\begin{array}{l}\text { UC Davis Lactation } \\
\text { Cohort }\end{array}$ & Yes & 95 & 60 & 60 \\
\hline $\begin{array}{l}\text { Cincinnati, } \\
\text { Ohio, United } \\
\text { States }\end{array}$ & No & PREVAIL & No & 245 & 45 & 26 \\
\hline
\end{tabular}




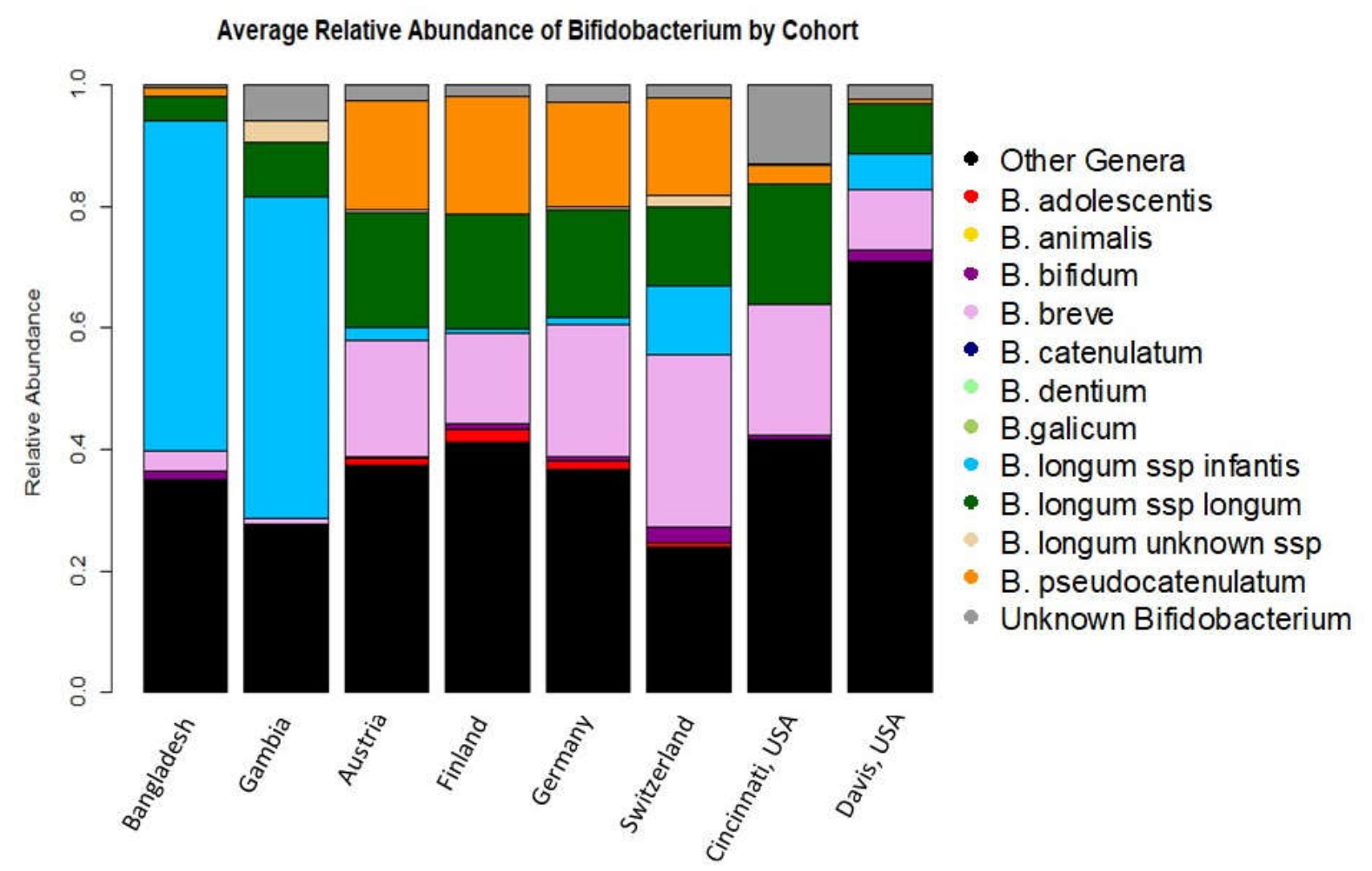

Figure 1: Average gut microbiome colonization patterns by country. 
Table 2: Summary of Bifidobacterium prevalence in infants aged 1 to 2 months. Except for information on breastfeeding initiation and duration in a cohort, values are based solely on infants who were breastfed. The breastfeeding initiation (ever breastfed) rate and breastfeeding duration were calculated using data from all infants in a cohort with an available sample and a known breastfeeding status.

\begin{tabular}{|c|c|c|c|c|c|c|c|c|c|c|c|}
\hline $\begin{array}{l}\text { Cohort } \\
\text { Location }\end{array}$ & $\begin{array}{c}\text { Ever } \\
\text { breastfe } \\
\text { d } \\
\text { (median } \\
\text { duration } \\
\text { ) }\end{array}$ & $\begin{array}{c}\text { Historic } \\
\text { breastfe } \\
\text { eding } \\
\text { pattern }\end{array}$ & $\begin{array}{c}\text { Any } \\
\text { Bifidobac } \\
\text { terium }\end{array}$ & $\begin{array}{c}\text { B. } \\
\text { adolesce } \\
\text { ntis }\end{array}$ & $\begin{array}{c}\text { B. } \\
\text { animali } \\
\mathrm{s}\end{array}$ & $\begin{array}{c}\text { B. } \\
\text { bifidu } \\
\text { m }\end{array}$ & $\begin{array}{c}\text { B. } \\
\text { breve }\end{array}$ & $\begin{array}{c}\text { B. longum } \\
\text { subsp } \\
\text { infantis }\end{array}$ & $\begin{array}{l}\text { B. longum } \\
\text { subsp } \\
\text { longum }\end{array}$ & $\begin{array}{c}\text { B. } \\
\text { longu } \\
\text { m } \\
\text { subsp } \\
\text { unkno } \\
\text { wn }\end{array}$ & $\begin{array}{c}\text { B. } \\
\text { pseudocaten } \\
\text { ulatum }\end{array}$ \\
\hline $\begin{array}{c}\text { Banglad } \\
\text { esh }\end{array}$ & $\begin{array}{c}100 \%(>2 \\
\text { years) }\end{array}$ & High & $100 \%$ & $5.8 \%$ & $2.6 \%$ & $32.5 \%$ & $\begin{array}{c}40.1 \\
\% \\
\end{array}$ & $83.6 \%$ & $26.3 \%$ & $1.1 \%$ & $8.4 \%$ \\
\hline Gambia & $\begin{array}{c}100 \% \\
\text { (Unkno } \\
\text { wn) }\end{array}$ & High & $100 \%$ & $0 \%$ & $0 \%$ & $0 \%$ & $25 \%$ & $91.7 \%$ & $54.2 \%$ & $12.5 \%$ & $0 \%$ \\
\hline Austria & $\begin{array}{c}91.8 \% \\
(6.8 \\
\text { months })\end{array}$ & Medium & $100 \%$ & $23.7 \%$ & $0.82 \%$ & $6.6 \%$ & $\begin{array}{c}55.7 \\
\%\end{array}$ & $4.1 \%$ & $61.5 \%$ & $4.9 \%$ & $52.4 \%$ \\
\hline Finland & $\begin{array}{l}99.3 \%(8 \\
\text { months) }\end{array}$ & Medium & $100 \%$ & $15.5 \%$ & $0 \%$ & $8.8 \%$ & $\begin{array}{c}40.0 \\
\% \\
\end{array}$ & $0.74 \%$ & $56.3 \%$ & $3.7 \%$ & $51.1 \%$ \\
\hline $\begin{array}{c}\text { German } \\
y\end{array}$ & $\begin{array}{c}91.1 \% \\
(7.4 \\
\text { months) } \\
\end{array}$ & Medium & $100 \%$ & $18.1 \%$ & $0.67 \%$ & $7.4 \%$ & $\begin{array}{c}61.1 \\
\%\end{array}$ & $4.0 \%$ & $61.7 \%$ & $4.0 \%$ & $57.0 \%$ \\
\hline $\begin{array}{c}\text { Switzerl } \\
\text { and }\end{array}$ & $\begin{array}{l}97.3 \%(8 \\
\text { months) }\end{array}$ & Medium & $100 \%$ & $4.8 \%$ & $0 \%$ & $11.1 \%$ & $\begin{array}{c}58.7 \\
\% \\
\end{array}$ & $14.8 \%$ & $41.8 \%$ & $6.3 \%$ & $49.7 \%$ \\
\hline $\begin{array}{c}\text { Davis, } \\
\text { Californi } \\
\text { a, } \\
\text { United } \\
\text { States } \\
\end{array}$ & $\begin{array}{c}100 \% \\
(9.3 \\
\text { months) }\end{array}$ & Low & $65 \%$ & $8.3 \%$ & $0 \%$ & $13.3 \%$ & $\begin{array}{c}36.7 \\
\%\end{array}$ & $8.3 \%$ & $36.7 \%$ & $1.7 \%$ & $15 \%$ \\
\hline $\begin{array}{c}\text { Cincinna } \\
\text { ti, Ohio, } \\
\text { United } \\
\text { States }\end{array}$ & $\begin{array}{c}86.5 \% \\
(3.1 \\
\text { months) }\end{array}$ & Low & $97 \%$ & $11.5 \%$ & $0 \%$ & $19.2 \%$ & $\begin{array}{c}61.5 \\
\%\end{array}$ & $0 \%$ & $69.2 \%$ & $0 \%$ & $19.2 \%$ \\
\hline
\end{tabular}


Table 3. Summary of GEE models comparing presence or absence of Bifidobacterium species in individual infants by cohort history of breastfeeding. Reference group was the high breastfeeding group. To account for multiple comparisons, a p-value less than 0.0062 is considered significant. The model for $B$. animalis failed to run, because after excluding infants who were not breastfed at time of sample collection, there were no infants from a low historical breastfeeding pattern cohort colonized by B. animalis.

\begin{tabular}{|c|c|c|c|c|c|c|c|c|}
\hline $\begin{array}{l}\text { Cohort } \\
\text { Breast- } \\
\text { feeding } \\
\text { pattern. }\end{array}$ & $\begin{array}{c}B . \\
\text { adolescent } \\
\text { is } \\
\text { Odds ratio } \\
\text { (95\% CI, } \\
\text { p-value) }\end{array}$ & $\begin{array}{c}B . \\
\text { animal } \\
\text { is } \\
\text { Odds } \\
\text { ratio } \\
(95 \% \\
\text { CI, p- } \\
\text { value) }\end{array}$ & $\begin{array}{c}B . \\
\text { bifidu } \\
m \\
\text { Odds } \\
\text { ratio } \\
(95 \% \\
\text { CI, p- } \\
\text { value) }\end{array}$ & $\begin{array}{c}\text { B. breve } \\
\text { Odds } \\
\text { ratio } \\
(95 \% \\
\text { CI, p- } \\
\text { value) }\end{array}$ & $\begin{array}{c}\text { B. longum } \\
\text { subspecie } \\
\text { s infantis } \\
\text { Odds } \\
\text { ratio (95\% } \\
\text { CI, p- } \\
\text { value) }\end{array}$ & $\begin{array}{l}\text { B. longum } \\
\text { subspecie } \\
\text { s longum } \\
\text { Odds } \\
\text { ratio (95\% } \\
\text { CI, p- } \\
\text { value) }\end{array}$ & $\begin{array}{c}\text { B. longum } \\
\text { unknown } \\
\text { subspecie } \\
\text { s } \\
\text { Odds } \\
\text { ratio } \\
\text { (95\% CI, } \\
\text { p-value) }\end{array}$ & $\begin{array}{c}\text { B. } \\
\text { pseudocatenul } \\
\text { atum } \\
\text { Odds ratio } \\
\text { (95\% CI, p- } \\
\text { value) }\end{array}$ \\
\hline $\begin{array}{l}\text { Mediu } \\
\mathrm{m}\end{array}$ & $\begin{array}{c}4.1(1.6- \\
11 \\
\mathrm{p}=0.0041)\end{array}$ & NA & $\begin{array}{c}0.22 \\
(0.16- \\
0.31, \\
p<0.00 \\
01)\end{array}$ & $\begin{array}{c}2.0(1.3 \\
-3.1 \\
\mathrm{p}=0.001 \\
9)\end{array}$ & $\begin{array}{c}0.010 \\
(0.0037- \\
0.029 \\
p<0.0001)\end{array}$ & $\begin{array}{c}2.2(0.98- \\
5.0 \\
\mathrm{p}=0.055)\end{array}$ & $\begin{array}{c}2.8(0.92- \\
8.2 \\
\mathrm{p}=0.069)\end{array}$ & $\begin{array}{c}12(10-13 \\
\mathrm{p}<0.0001)\end{array}$ \\
\hline Low & $\begin{array}{c}2.4(1.0- \\
5.6, \\
\mathrm{p}=0.041)\end{array}$ & NA & $\begin{array}{c}0.40 \\
(0.28- \\
0.58 \\
p<0.00 \\
01)\end{array}$ & $\begin{array}{c}1.4 \\
(0.70- \\
2.9 \\
\mathrm{p}=0.32)\end{array}$ & $\begin{array}{c}0.0084 \\
(0.0024- \\
0.029 \\
p<0.0001)\end{array}$ & $\begin{array}{c}1.8(0.57- \\
5.7 \\
\mathrm{p}=0.31)\end{array}$ & $\begin{array}{c}0.63(0.16 \\
-2.4 \\
p=0.51)\end{array}$ & $\begin{array}{c}2.1(1.8-2.5 \\
\mathrm{p}<0.0001)\end{array}$ \\
\hline
\end{tabular}

Table 4. Table of number of European infants colonized at any level by B. longum subspecies infantis at age 1 year by breastfeeding status. Prevalence of B. longum subspecies infantis appears to decrease rapidly during the weaning period, supporting the assumption that vertical transmission will be rare. P-values are for the Chi-square tests comparing B. longum subsp. infantis detection in still breastfed vs. no longer breastfed infants.

\begin{tabular}{|c|c|c|c|c|}
\hline Country & $\begin{array}{l}\text { Breast Fed at } 1 \text { Year } \\
\text { (European } \\
\text { countries) or } 2 \\
\text { Years (Bangladesh) }\end{array}$ & $\begin{array}{c}\text { B. longum } \\
\text { subspecies infantis } \\
\text { detected }\end{array}$ & $\begin{array}{c}\text { B. longum } \\
\text { subspecies infantis } \\
\text { NOT detected }\end{array}$ & $\begin{array}{c}\text { Percentage of } \\
\text { infants colonized }\end{array}$ \\
\hline \multirow{2}{*}{$\begin{array}{l}\text { Austria } \\
(p=0.003)\end{array}$} & Yes & 5 & 14 & $36 \%$ \\
\hline & No & 2 & 70 & $2.8 \%$ \\
\hline \multirow{2}{*}{$\begin{array}{l}\text { Finland } \\
(\mathrm{p}=0.65)\end{array}$} & Yes & 1 & 37 & $2.6 \%$ \\
\hline & No & 0 & 91 & $0 \%$ \\
\hline \multirow{2}{*}{$\begin{array}{l}\text { Germany } \\
\qquad(p=1)\end{array}$} & Yes & 1 & 18 & $5.3 \%$ \\
\hline & No & 2 & 89 & $2.2 \%$ \\
\hline
\end{tabular}




\begin{tabular}{ccccc}
\hline $\begin{array}{c}\text { Switzerland } \\
(\mathrm{p}<0.0001)\end{array}$ & Yes & 10 & 24 & $29 \%$ \\
\cline { 2 - 5 } & No & 6 & 135 & $4.3 \%$ \\
\hline $\begin{array}{c}\text { Bangladesh } \\
(\mathrm{p}=0.35)\end{array}$ & Yes & 13 & 51 & $20.3 \%$ \\
\cline { 2 - 5 } & No & 5 & 40 & $11.1 \%$ \\
\hline
\end{tabular}
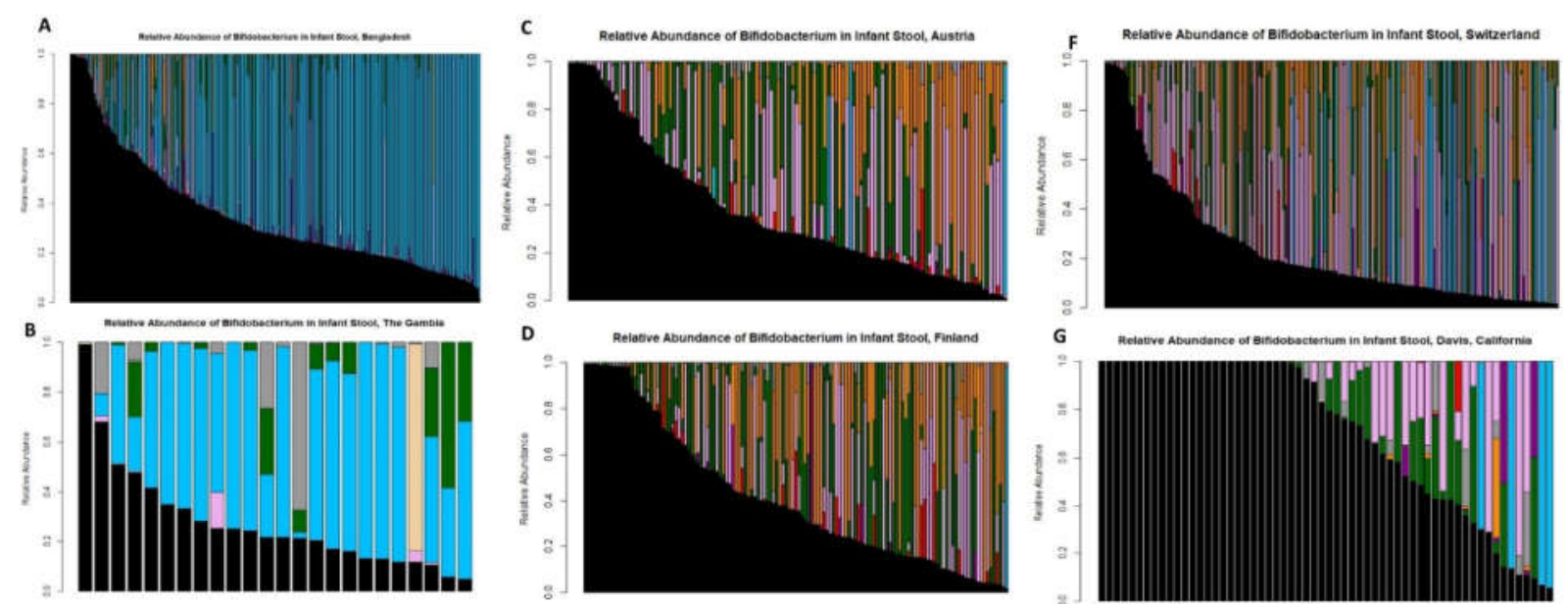

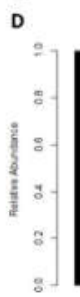

Reliatve Abundance of Bindobacterium in intant stoot. Finiand

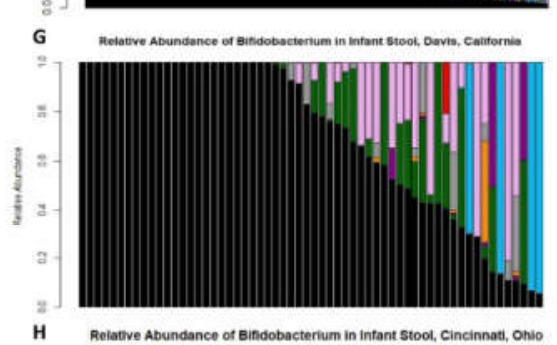

- Other Genera

- B. adolescentis

B. animalis

- B. bifidum

B. breve

- B catenulatum

- B. dentium

B.galicum

- B. longum ssp infantis

- B. longum ssp longum

B. longum unknown ssp

- B. pseudocatenulatum

- Unknown Bifidobacterium
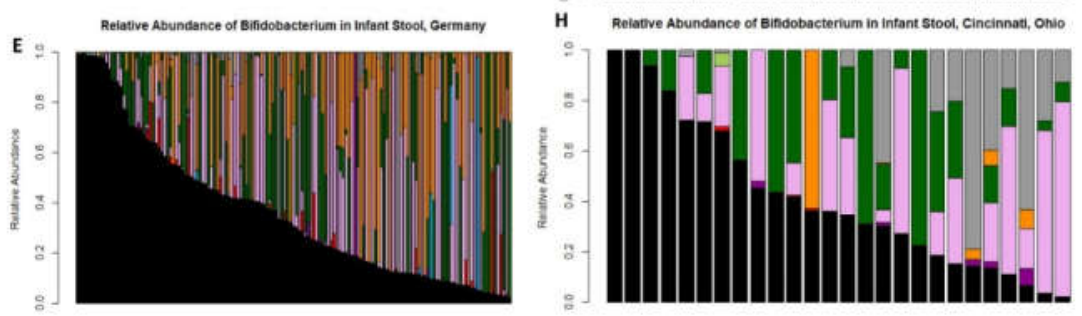

Figure 2. Relative abundance of different Bifidobacterium species in infant stool. $B$. longum ssp infantis is in blue, non-Bifidobacterium taxa are black. (A) Bangladesh (B) The Gambia (C) Austria (D) Finland (E) Germany (F) Switzerland (G) Davis, CA, USA (H) Cincinnati, $\mathrm{OH}$, USA. 


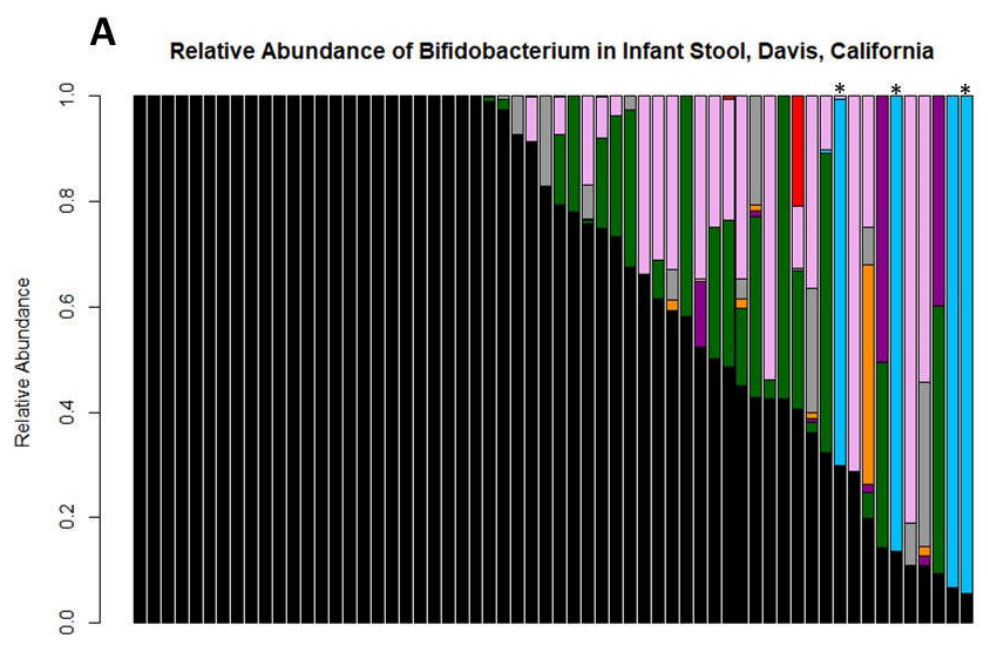

B Bifidobacterium Relative Abundance in Davis at Age 3 Days

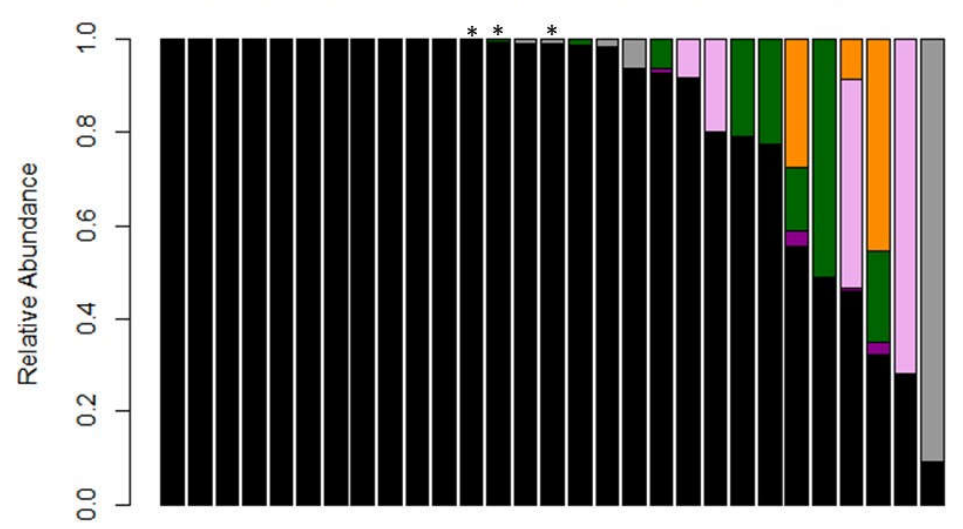

C Bifidobacterium Relative Abundance in Davis at Age 1 Month

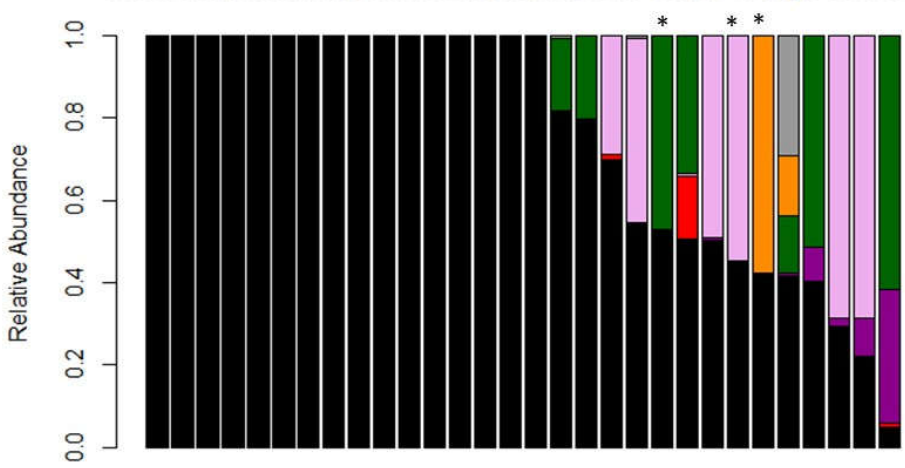

- Other Genera

- B. adolescentis

- B. animalis

- B. bifidum

- B. breve

- B. catenulatum
B. dentium
- B.galicum
- B. longum ssp infantis
- B. longum ssp longum
B. longum unknown ssp
- B.pseudocatenulatum
- Unknown Bifidobacterium

Figure 3. Bifidobacterium colonization in Davis, CA infants over time. Stars indicate samples from infants who were colonized by B. longum subsp. infantis at age 2 months. (A) Age 2 months samples (repeated from Figure 2) (B) Age 3 Days (C) Age 1 Month. 
Bifidobacterium in Stool in Children Age 2 Years, Bangladesh

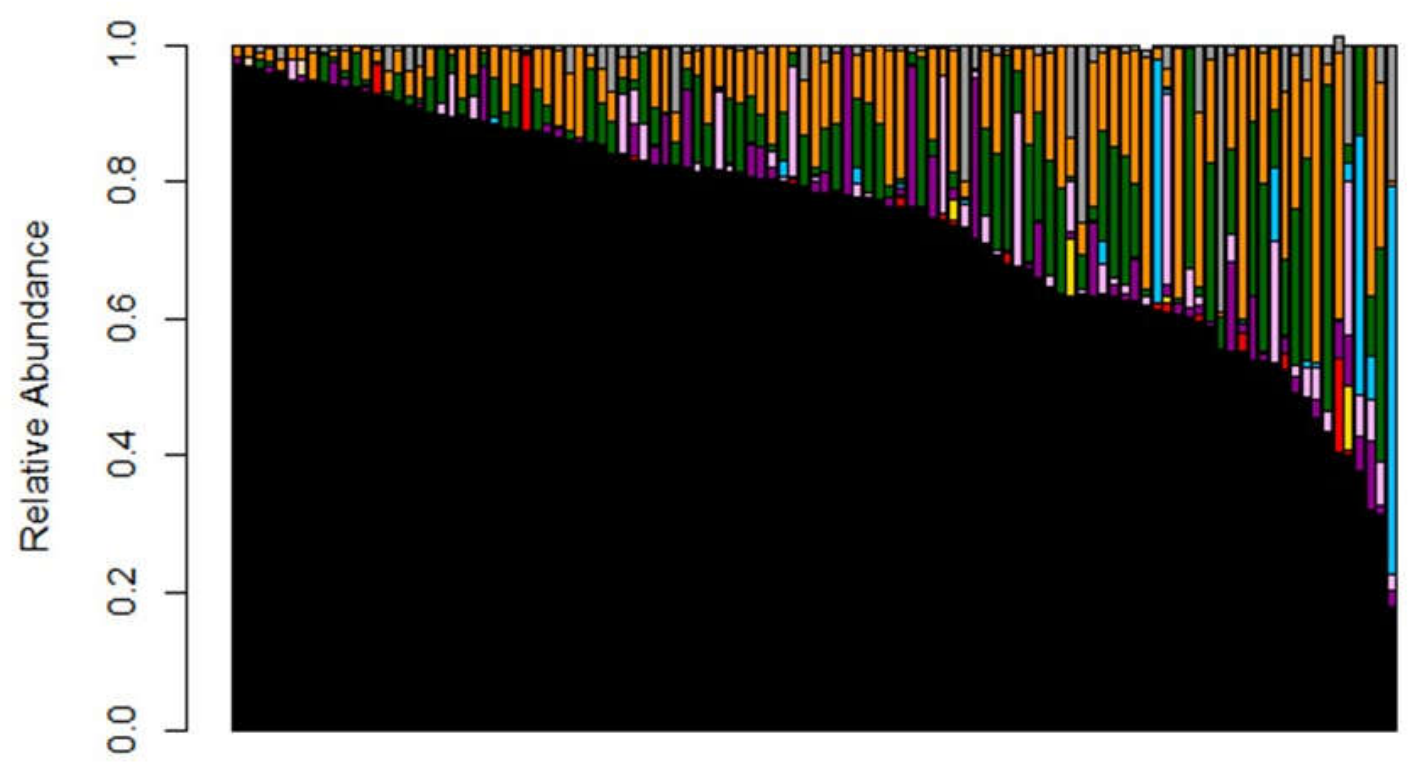

Figure 4. Bifidobacterium colonization in Bangladeshi cohort in infants age 2 years. Slight deviations from $100 \%$ are related to rounding errors in the Bif-TRFLP calculation of percentages. Note the reduced prevalence of $B$. longum subspecies infantis in blue; this is a subset of the same infants shown in Figure 2B. 


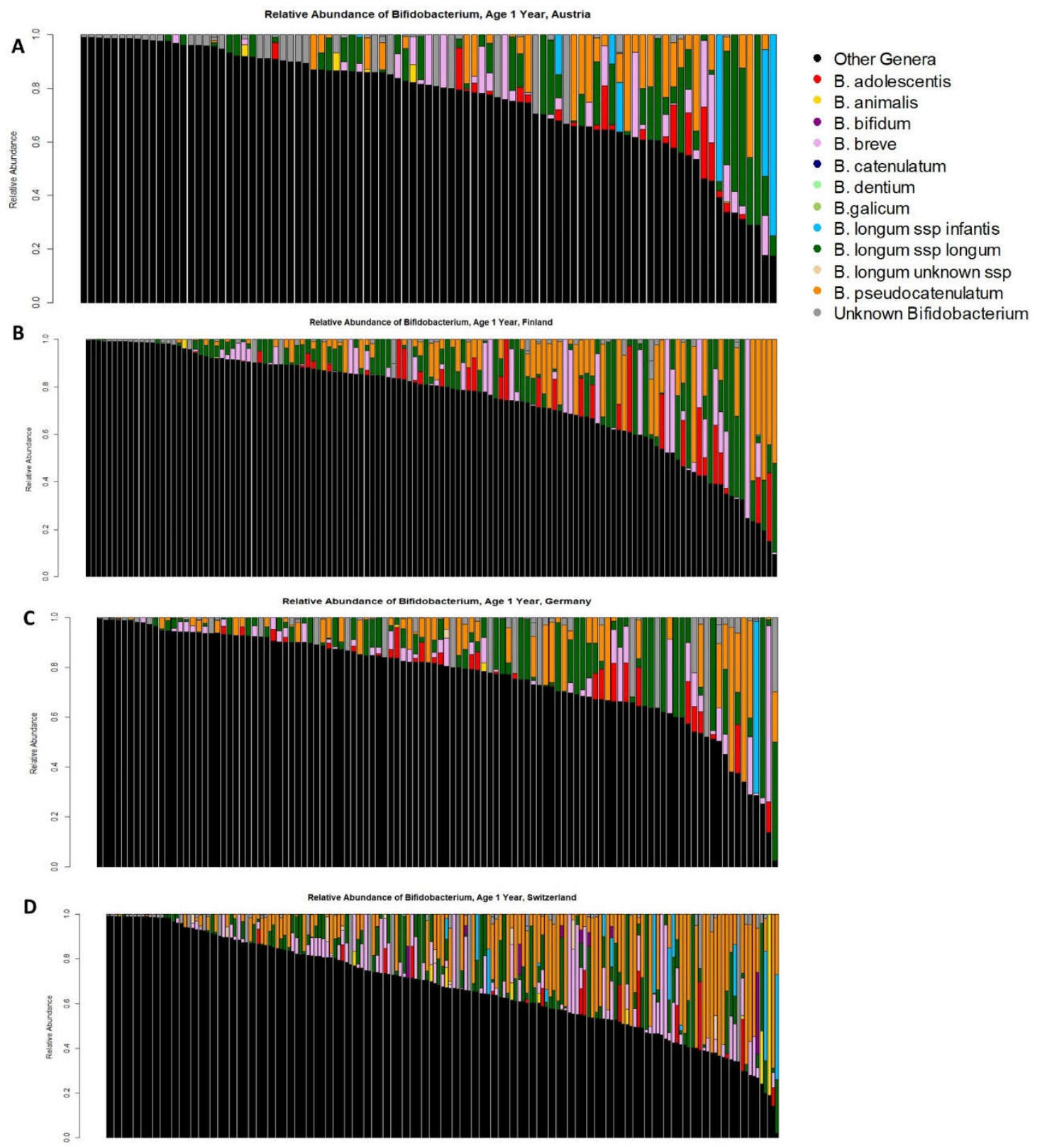

Figure 5. Bifidobacterium colonization in infants at age 1 year in (A) Austria (B) Finland (C) Germany and (D) Switzerland. 